\title{
Theoretical model for modal dispersal of polarization information and its recovery by phase conjugation
}

\author{
Amnon Yariv, Yasuo Tomita, and Kazuo Kyuma* \\ California Institute of Technology, Pasadena, California 91125
}

Received May 27, 1986; accepted September 4, 1986

\begin{abstract}
A theoretical model is proposed for explaining the recently observed modal dispersal of polarization information and its recovery in an experiment in which the coupling to the phase conjugator is by means of a (multi)mode dispersing fiber.
\end{abstract}

In a recent paper ${ }^{1}$ we reported on a series of experiments in which an optical field of arbitrary polarization regains its original state of polarization following phase conjugation by virtue of modal scrambling in a fiber placed in front of the phase-conjugate mirror (PCM). This happens in spite of the fact that the PCM reflects only one transverse component, $x$ or $y$, of the incident field.

The relevant geometry and the experimental setup are shown in Fig. 1. An incident plane wave is imparted an arbitrary state of elliptic polarization by means of a retarding quarter-wave $(\lambda / 4)$ plate. It is next launched into a multimode polarization- and modalscrambling fiber. $\mathrm{A} \mathrm{BaTiO}_{3}$ passive (PCM) ${ }^{2,3}$ reflects only the $x$ (or $y$ ) component of the field. At the input end of the fiber the left-traveling returning field is found to be a true phase-conjugate replica of the input field including both polarizations. A more detailed description of the experiment and the observations are included in Ref: 1 . Our basic model makes use of the observation that the fiber causes strong intermodal scattering so that power coupled into any one mode at the input is distributed essentially uniformly among all the other modes including these of orthogonal polarization.

Referring to Fig. 1, we write the right-traveling field at the input plane (1) to the fiber as

$$
\begin{aligned}
\mathbf{E}^{(1)} & =\sum_{n=1}^{\mathrm{N}}\left[a_{x n}{ }^{(1)} \mathbf{e}_{x n}+a_{y n}{ }^{(1)} \mathbf{e}_{y n}\right] \\
& \equiv\left[\begin{array}{c}
a_{x l}{ }^{(1)} \\
\vdots \\
a_{x N}{ }^{(1)} \\
a_{y l}{ }^{(1)} \\
\vdots \\
a_{y N}{ }^{(1)}
\end{array}\right] \equiv\left[\begin{array}{c}
A_{x}^{(1)} \\
A_{y}^{(1)}
\end{array}\right],
\end{aligned}
$$

where $\mathbf{e}_{x n}$ is the $n$th transverse fiber mode that is predominantly $x$ polarized, while $\mathbf{e}_{y n}$ is $y$ polarized. $A_{x}^{(1)}$ and $A_{y}^{(1)}$ are column vectors of rank $N$ whose elements are the complex amplitudes $a_{x n}{ }^{(1)}$ and $a_{y n}{ }^{(1)}$, respectively. The propagation from left to right through the fiber including intermodal coupling can be represented by the matrix relation

$$
\mathbf{E}^{(2)}=M \mathbf{E}^{(1)}
$$

and

$$
M=\left(\begin{array}{ll}
M_{x x} & M_{x y} \\
M_{y x} & M_{y y}
\end{array}\right),
$$

where $M_{i j}$ is an $N \times N$ matrix. The field $\mathbf{E}^{(2)}$ is shorn of its $y$ component by the polarizing beam splitter so that the field returning to the fiber following phase conjugation is $x$ polarized. The field $\mathbf{E}^{(3)}$ reentering the fiber can thus be expressed as

$$
\mathbf{E}^{(3)}=R C\left[\mathbf{E}^{(2)}\right]^{*},
$$

where $R$ is the PCM reflectivity and the matrix $C$, representing the removal of the $y$ polarization, is

$$
C=\left(\begin{array}{ll}
I & 0 \\
0 & 0
\end{array}\right)
$$

where $I$ is an $N \times N$ unit matrix.

The output field $\mathbf{E}^{(4)}$ leaving the fiber from the input face is

$$
\mathbf{E}^{(4)}=M^{\prime} \mathbf{E}^{(3)}=R M^{\prime} C M^{*}\left[\mathbf{E}^{(1)}\right]^{*},
$$

where $M^{\prime}$ describes the propagation in the fiber from right to left.

In what follows we show that the matrix product $M^{\prime} C M^{*}$ is proportional to the unit matrix so that

$$
\mathbf{E}^{(4)}=R\left[\mathbf{E}^{(1)}\right]^{*},
$$

which corresponds to polarization-preserving phase conjugation of the input field $\mathbf{E}^{(1)}$.

In a fiber with no loss the total power in the mode ensemble is conserved so that

$$
\sum_{n=1}^{N}\left[\left|a_{x n}{ }^{(1)}\right|^{2}+\left|a_{y n}{ }^{(1)}\right|^{2}\right]=\sum_{n=1}^{N}\left[\left|a_{x n}{ }^{(2)}\right|^{2}+\left|a_{y n}{ }^{(2)}\right|^{2}\right]
$$

Using Eq. (2), the last relation is satisfied if and only if 


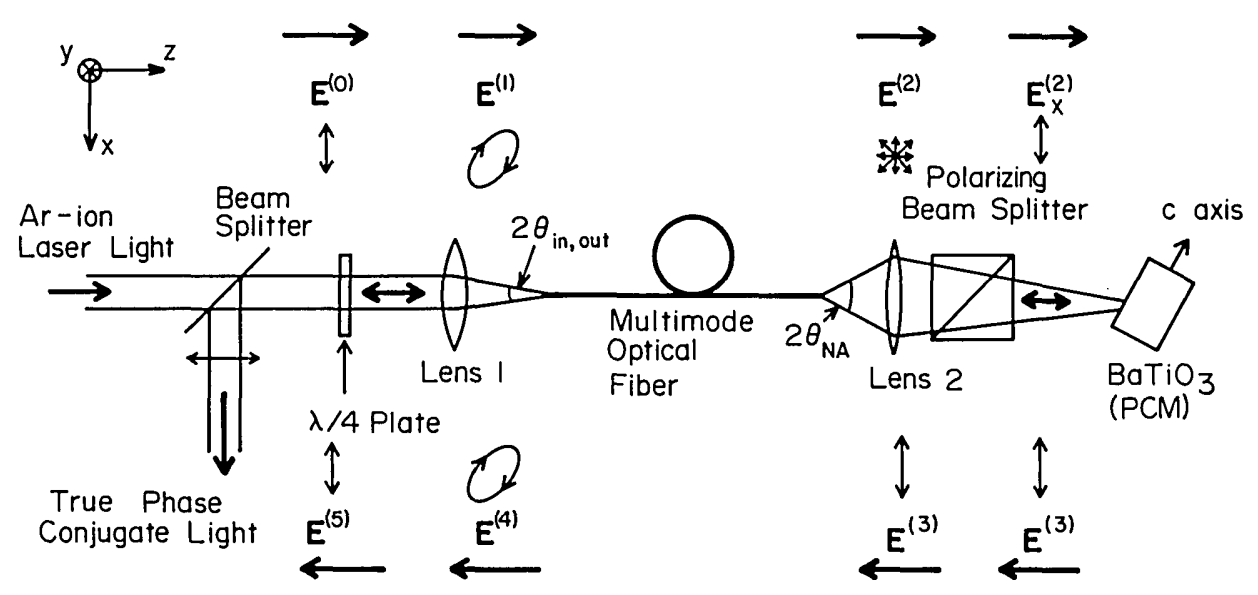

Fig. 1. Experimental arrangement for polarization recovery in phase conjugation using polarization- and modal-scrambling multimode fiber.

$$
M^{\dagger}=M^{-1} \text {, }
$$

where $M^{\dagger}$ is the Hermitian adjoint of $M$. Time-reversal symmetry requires that propagation through the fiber from left to right followed by phase conjugation and reverse propagation from right to left result in a field that is the complex conjugate of the original. In our matrix language this translates to

$$
M^{\prime} M^{*}=I \text {. }
$$

Combining Eqs. (7) and (8), we obtain

$$
M^{\prime}=\left(M^{*}\right)^{-1}=\left(M^{-1}\right)^{*}=\left(M^{\dagger}\right)^{*}=M_{t},
$$

where $M_{t}$ is the transpose of $M$.

The assumption leading to Eq. (8) may be shown also to result in the relation $M_{i j}=M_{j i}$ and from it $M=$ $M^{\prime}$. For our purposes here Eq. (9) is sufficient. In terms of the submatrices appearing in Eq. (3) the relation $M^{\prime}=M_{t}$ becomes

$$
\begin{aligned}
& \left(M^{\prime}{ }_{x x}\right)_{i j}=\left(M_{x x}\right)_{j i}, \\
& \left(M^{\prime}{ }_{y y}\right)_{i j}=\left(M_{y y}\right)_{j i}, \\
& \left(M_{x y}^{\prime}\right)_{i j}=\left(M_{y x}\right)_{i j}, \\
& \left(M^{\prime}{ }_{y x}\right)_{i j}=\left(M_{x y}\right)_{j i} .
\end{aligned}
$$

The experiments indicate that the fiber causes complete depolarization of any input field as well as near equipartition of any modal energy among the ensemble of modes so that

$$
M_{i j}=\frac{1}{\sqrt{2 N}} \exp \left(i \phi_{i j}\right)
$$

where the $\phi_{i j}$ are distributed essentially uniformly over the $-\pi-+\pi$ interval.

We can now evaluate the matrix $M^{\prime} C M^{*}$. Using Eqs. (3) and (5) we get

$$
M^{\prime} C M^{*}=\left(\begin{array}{cc}
M^{\prime}{ }_{x x} M^{*}{ }_{x x} & M^{\prime}{ }_{x x} M^{*}{ }_{x y} \\
M^{\prime}{ }_{y x} M^{*}{ }_{x x} & M^{\prime}{ }_{y x} M^{*}{ }_{x y}
\end{array}\right)
$$

and

$$
\begin{aligned}
\left(M_{x x}^{\prime} M^{*}{ }_{x x}\right)_{i j} & =\left(M^{\prime}{ }_{x x}\right)_{i k}\left(M_{x x}\right)^{*}{ }_{k j} \\
& =\left(M_{x x}\right)_{k i}\left(M_{x x}\right)^{*}{ }_{k j} \\
& =\frac{1}{2 N} \sum_{k=1}^{N} \exp \left\{i\left[\left(\phi_{x x}\right)_{k i}-\left(\phi_{x x}\right)_{k j}\right]\right\} \\
& = \begin{cases}1 / 2 & \text { for } i=j, \\
O(1 / N) & \text { for } i \neq j,\end{cases}
\end{aligned}
$$

where we sum over repeated indices and make use of Eqs. (10) and (11). The $N^{-1}$ in Eq. (13b) is due to the random-walk nature represented by the summation of the phasors $\exp \left\{i\left[\left(\phi_{x x}\right)_{k i}-\left(\phi_{x x}\right)_{k j}\right]\right\}$.

In a similar fashion we find

$$
\begin{aligned}
\left(M^{\prime}{ }_{y x} M^{*}{ }_{x y}\right)_{i j} & = \begin{cases}1 / 2 & \text { for } i=j, \\
O(1 / N) & \text { for } i \neq j,\end{cases} \\
\left(M^{\prime}{ }_{x x} M^{*}{ }_{x y}\right)_{i j} & =O(1 / N), \\
\left(M^{\prime}{ }_{y x} M^{*}{ }_{x x}\right)_{i j} & =O(1 / N) .
\end{aligned}
$$

For a large number of modes $N$ we thus find that to a high degree of accuracy the round-trip field matrix $M^{\prime} C M$ is proportional to the $2 N \times 2 N$ unit matrix

$$
M^{\prime} C M^{*} \approx \frac{1}{2}\left(\begin{array}{ll}
I & 0 \\
0 & I
\end{array}\right)
$$

and

$$
\mathbf{E}^{(4)}=\frac{1}{2} R\left[\mathbf{E}^{(1)}\right]^{*}
$$

constituting full phase conjugation of the vector $\left[\mathbf{E}^{(1)}\right]$ including both orthogonal polarizations.

In summary, we have developed a theoretical model that explains recently observed phenomena in which strong intermodal coupling in a fiber is used to store polarization information, thereby permitting a reconstruction of the original state of polarization of a field 
following the elimination of one transverse component $(x$ or $y$ ) and the phase conjugation of the remaining ( $y$ or $x$ ) component. In some basic sense the phenomenon is reminiscent of holography, in which an image can be reconstructed in spite of elimination of part of the hologram. The difference is that here information is distributed not throughout the physical space (as in a hologram) but throughout the modal space and does not involve a physical modification of a storage medium. The reconstruction of the original state from the information stored in the surviving modes depends on the existence of a large number of modes in the ensemble so that the undesired terms average out to zero. We anticipate that this new mode of information handling will find numerous new applications.
The authors are grateful to the U.S. Army Research Office (R. Guenther) and the U.S. Air Force Office of Scientific Research (H. Schlossberg) for support of this work.

* On leave from Central Research Laboratory, Mitsubishi Electric Corporation, 8-1-1 Tsukaguchi-Honmachi, Amagasaki, Hyogo, 661 Japan.

\section{References}

1. K. Kyuma, A. Yariv, and S. K. Kwong, Appl. Phys. Lett. 49, 617 (1986).

2. M. Cronin-Golomb, B. Fisher, J. O. White, and A. Yariv, IEEE J. Quantum Electron. QE-20, 12 (1984).

3. J. Feinberg, Opt. Lett. 7, 486 (1982). 